\title{
Demographic and Aetiological Profile of Microbial Corneal Ulcer in a Hill State in North India
}

\author{
Chintan Malhotra, Ramesh Chander Nagpal, Ribhu Soni, Harsh Bahadur \\ Department of Ophthalmology, Himalayan Institute of Medical Sciences, Dehradun, India
}

\begin{abstract}
Aim: To ascertain the demographic and aetiological profile of microbial corneal ulcer in Uttarakhand, a predominantly hilly state of North India.

Methods: In this prospective non-randomized study, 180 consecutive patients with suspected microbial corneal ulcer attending the ophthalmology outpatient department of the Himalayan Institute of Medical Sciences, Dehradun, India, were evaluated. Corneal scrapings were taken and subjected to microbiological analysis, including Gram stain, potassium hydroxide wet mount, and bacterial and fungal culture.

Results: Among 180 patients, male sex, prior history of ocular trauma with vegetative materials, and occupation involving outdoor activity, especially agricultural work, were the major risk factors for development of corneal ulcer in the population studied. Forty percent of the ulcers were fungal, $28 \%$ were bacterial, 10\% showed mixed bacterial and fungal growth, and 5\% were viral. The aetiology could not be determined in $17 \%$ of patients. Fusarium spp was the most common fungal isolate (87.5\%) while Staphylococcus aureus was the commonest bacterial isolate (53\%).

Conclusions: Fungal ulcers, especially due to Fusarium spp, were the most common type, followed by bacterial ulcers due to Gram-positive bacteria, especially S aureus. This information may be useful for empirical treatment of patients with corneal ulcer in remote areas, where microbiological diagnostic facilities are often not available.
\end{abstract}

Key words: Corneal ulcer, Keratitis, Fusarium, Staphylococcus aureus

Asian J Ophthalmol. 2011;12:211-5.

\section{Introduction}

Corneal ulceration in developing countries has been recognised as a silent epidemic. ${ }^{1} \mathrm{~A}$ previous report on the causes of blindness worldwide listed corneal scarring second only to cataract as the major aetiology of blindness and visual disability in many of the developing nations in Asia, Africa, and the Middle East. ${ }^{2}$

Corneal ulcer is characterised by an epithelial defect with infiltration of underlying and surrounding stroma. Corneal ulcer is an ocular emergency that requires prompt and appropriate management to ensure the best visual outcome. Corneal ulcers can be caused by trauma and other means of epithelial loss, including contact lens wear. Microbial organisms causing corneal ulcer include bacteria, fungi, parasites (Acanthamoeba), and viruses (herpes simplex, herpes zoster). Some of the common bacteria leading to corneal ulcer include Gram-positive cocci

Correspondence: Dr Chintan Malhotra, Department of Ophthalmology, Himalayan Institute of Medical Sciences, Swami Ram Nagar, Dehradun, Uttarakhand, India.

Tel: (91 135) 247 1568;

E-mail: drchintansingh@yahoo.co.in
(Staphylococcus epidermidis, Staphylococcus aureus, and Streptococcus pneumoniae), Gram-positive bacilli (Corynebacterium spp, Bacillus spp) and Gram-negative bacilli (Pseudomonas spp, Enterobacteriaceae spp, Haemophilus spp). The common fungi include Fusarium spp, Aspergillus spp, and Candida spp.

Two laboratory procedures are usually used to determine the aetiological agent causing the infectious keratitis: microscopy for direct visualisation of the organisms (using a variety of stains) and culture by inoculation under appropriate conditions to allow multiplication of organisms. Studies conducted in different parts of the world and in different regions of India showed varying microbiological profiles of the causative organism of infective keratitis. ${ }^{3-10}$ Knowledge of local aetiology within a region is of value for the management of suppurative keratitis in the event that microscopy and culture cannot be performed. ${ }^{10}$ Most of the population of Uttarakhand live in remote hilly areas where access to diagnostic facilities is not easily available. This study was conducted to ascertain the demographic and aetiological profile of corneal ulcer in the hill state of Uttarakhand in North India. 


\section{Methods}

\section{Patients}

All patients diagnosed with microbial corneal ulceration on the basis of symptoms and clinical examination who attended the ophthalmology outpatient department of the Himalayan Institute of Medical Sciences, Dehradun, India, from August 2008 to September 2009 were included in the study. All patients were examined according to a standardised format developed for the purpose after having obtained informed written consent.

\section{Design}

A detailed history, including mode of injury (if any), duration of injury, and any medications already taken, was obtained from each patient. Slit-lamp examination was done to note the clinical characteristics of the ulcer, including the site, size, shape, depth, margins, floor, surface, and vascularisation. Corneal sensitivity was tested and fluorescein staining of the cornea was done. Corneal ulcers were graded as non-severe or severe depending on the depth of the ulcer, presence and extent of suppuration, likelihood of perforation, and presence of scleral suppuration. ${ }^{11}$

Corneal scrapings were taken to determine the microbial organism causing the ulcer. The material obtained was subjected to Gram staining and potassium hydroxide $(\mathrm{KOH})$ wet mount preparation for microscopy and was also directly inoculated into culture media in the outpatients department. Bacterial culture was done using blood agar and MacConkey agar. Fungal culture was done on Sabouraud dextrose agar and brain heart infusion broth.

\section{Statistical Analysis}

Data were collected and entered into Epi Info for the Statistical Package for the Social Sciences (SPSS Inc, Chicago, USA). Chisquared test was used to determine the level of significance, and the strength of association between different variables was tested using odds ratios (OR) with $95 \%$ confidence intervals $(\mathrm{Cl})$. p Values of $<0.05$ were considered significant.

\section{Results}

180 consecutive patients with microbial corneal ulceration were enrolled. There were 144 men and 36 women, for a male to female ratio of $4: 1\left(\chi^{2}, 127.21 ; \mathrm{df}, 1 ; p<0.0001\right)$. The mean age was 42.5 years (SD, 16.0 years, range, 14-75 years). The largest age group was 30 to 39 years (Figure 1). Severe corneal ulcers were seen in 51 patients (28.3\%), while 129 (71.7\%) had non-severe ulcers.

Ocular trauma was the commonest predisposing factor leading to the development of corneal ulcer in 132 patients $(73.3 \%$; $\chi^{2}$, 304.0; df, 3; $p<0.0001 ; 0 R, 2.75 ; 95 \%$ Cl, 1.83-4.14). Other predisposing factors included prior cataract surgery $(n=24 ; 13.3 \%)$ and lagophthalmos $(n=6 ; 3.3 \%)$, while no underlying cause could be detected for 18 patients (10\%) [Table 1].

Agriculture was the most common occupation associated with risk of development of corneal ulceration (Table 1). Eighty four patients $(46.7 \%)$ were agricultural workers $\left(\chi^{2}, 63.47\right.$; df, 3 ; $\mathrm{p}<0.001 ; 0 \mathrm{R}, 2.15 ; 95 \% \mathrm{Cl}, 1.37-3.40), 66$ (78.6\%) of whom had ocular trauma with a sugarcane leaf prior to development of the corneal ulcer $\left(\chi^{2}, 54.86\right.$; df, $\left.1 ; p<0.0001\right)$; other causes of injury

Figure 1. Age of patients with microbial keratitis.

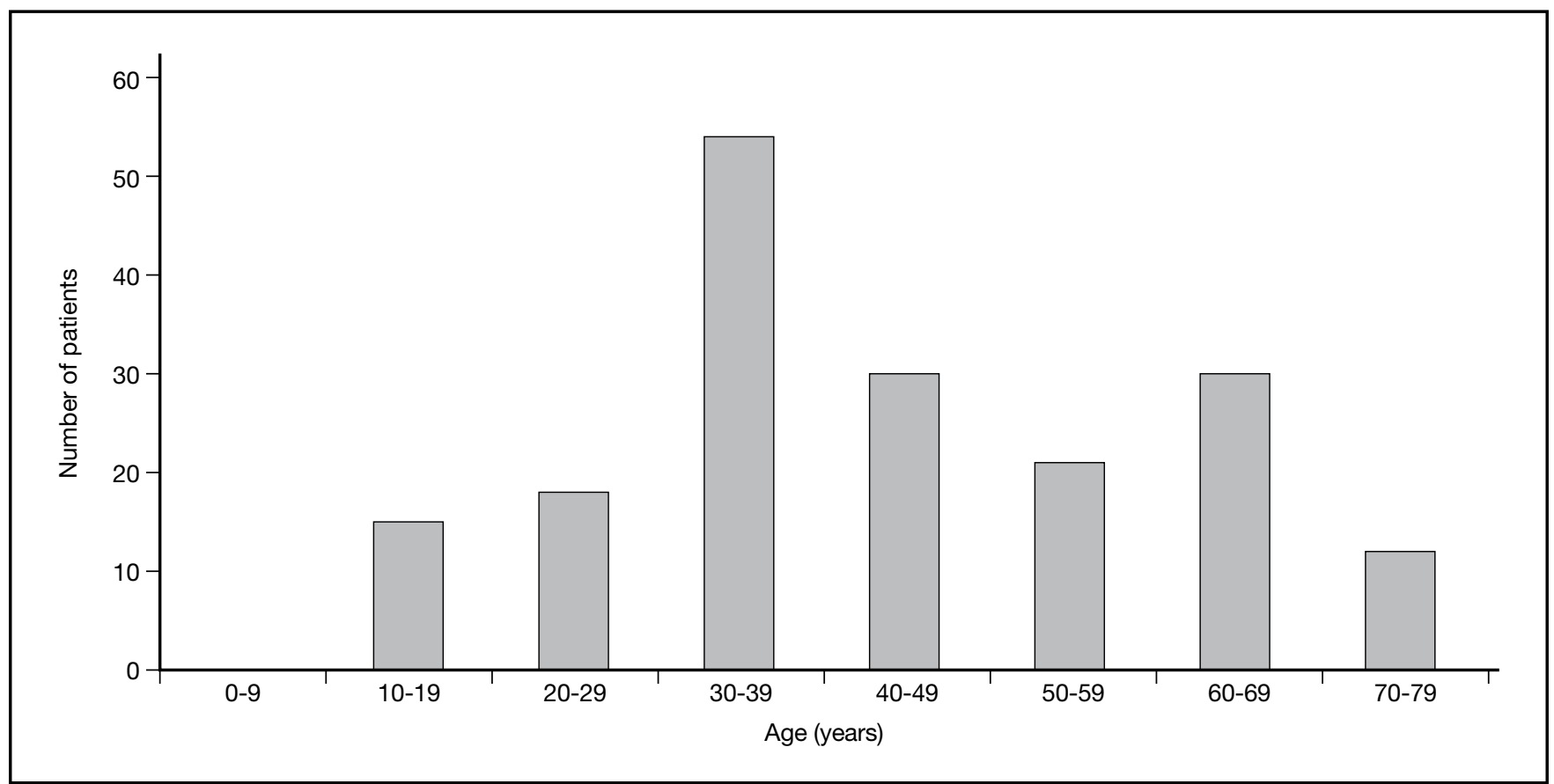


Table 1. Determinants of corneal ulceration among the study population $(n=180)$.

\begin{tabular}{|c|c|c|c|c|c|}
\hline \multicolumn{2}{|c|}{ Predisposing factors } & \multicolumn{2}{|c|}{ Occupation } & \multicolumn{2}{|c|}{ Seasonal variation } \\
\hline Factor & Number (\%) & Factor & Number (\%) & Factor & Number (\%) \\
\hline Trauma & $132(73.3)^{\star}$ & Agriculture & $84(46.7)^{\dagger}$ & January-March & $21(11.7)$ \\
\hline Cataract surgery & $24(13.3)$ & Industrial work & $39(21.7)$ & April-June & $27(15.0)$ \\
\hline Lagophthalmos & $6(3.3)$ & Household work & $33(18.3)$ & July-September & $81(45.0)^{\ddagger}$ \\
\hline Unknown & $18(10.0)$ & Other & 24 (13.3) & October-December & $51(28.3)$ \\
\hline
\end{tabular}

${ }^{*} \mathrm{p}<0.0001$ ( $\chi^{2}$ test); odds ratio, $2.75 ; 95 \%$ confidence interval, 1.83-4.10.

${ }^{\dagger} p<0.001$ ( $\chi^{2}$ test); odds ratio, $2.15 ; 95 \%$ confidence interval, $1.37-3.40$.

$\neq p<0.05$ ( $\chi^{2}$ test).

among agricultural workers were rice husk ( $n=5 ; 5.9 \%)$, fertilizer $(n=3 ; 3.6 \%)$, and branches of plants $(n=10 ; 11.9 \%)$.

Significant seasonal variation was seen, with the maximum number of corneal ulcerations ( $n=81 ; 45.0 \%$ ) occurring in the hot and humid period from July to September and the minimum $(\mathrm{n}=21 ; 11.7 \%)$ occurring in the colder months of January to March $\left(\chi^{2}, 66.13 ; \mathrm{df}, 3 ; p<0.05\right)$ [Table 1].

Corneal scraping and microbial analysis (microscopy and culture) was performed for 171 patients (95.0\%). Corneal specimens of 9 patients $(5.0 \%)$ who were diagnosed with viral keratitis because of the clinical signs were not sent for microbiological investigation. Of the 171 specimens sent for microbial analysis, $141(82.5 \%)$ were culture positive $\left(\chi^{2}, 144.1 ; \mathrm{df}, 1 ; p<0.001\right)$, while the remaining $30(17.5 \%)$ were culture negative. Amongst the culture-positive specimens, pure bacterial growth was seen in $51(36.2 \%)$, pure fungal growth in $72(51.1 \%)$, and mixed bacterial and fungal growth in $18(12.8 \%)$. Gram stain was positive for 39

Figure 2. Microbiological agents causing corneal ulcer.

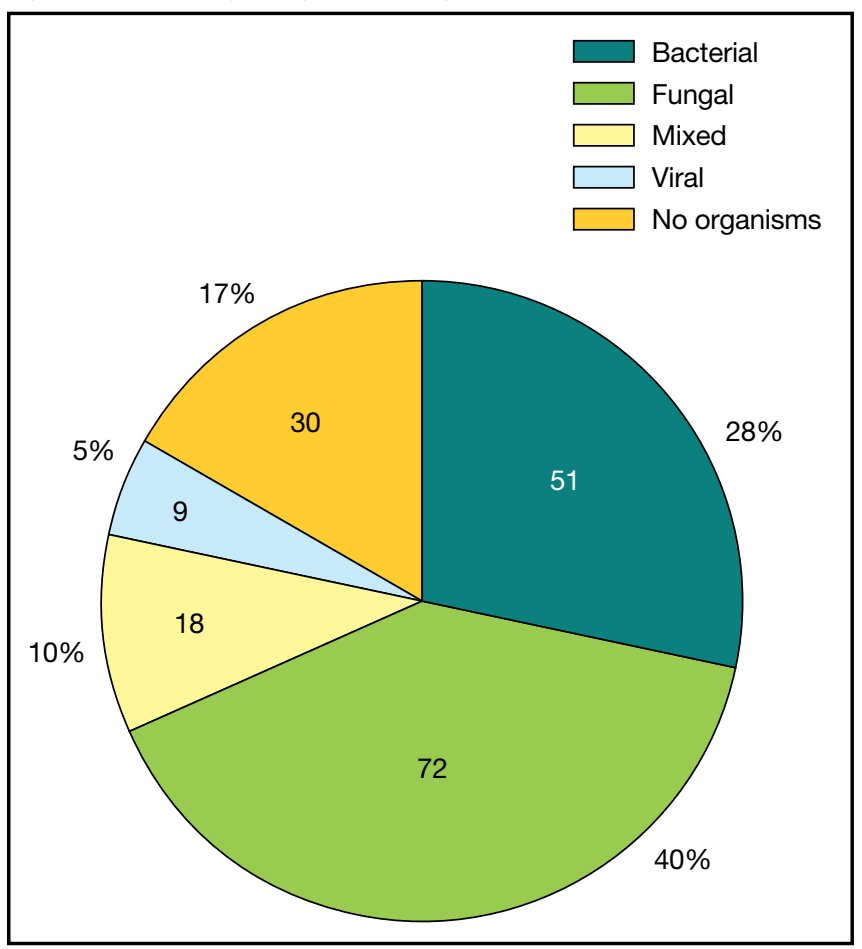

specimens $(76.5 \%)$ showing bacterial growth on culture $(p=0.001)$, while $\mathrm{KOH}$ wet mount was positive for 45 specimens $(62.5 \%)$ showing fungal culture. The sensitivity and specificity of Gram stain was $76 \%$ and $75 \%$, respectively, and of $\mathrm{KOH}$ wet mount was $67 \%$ and $68 \%$, respectively. None of the patients with a negative culture $(\mathrm{n}=30)$ had a positive Gram stain or $\mathrm{KOH}$ wet mount. Figure 2 shows the microbial aetiology.

Most of the bacterial isolates ( $\mathrm{n}=39 ; 76.5 \%)$ were Gram positive $\left(\chi^{2}, 28.59 ; \mathrm{df}, 1 ; \mathrm{p}<0.001\right)$. $S$ aureus was the predominant bacterial pathogen $\left(n=27 ; 52.9 \% ; \chi^{2}, 30.82 ; d f, 3 ; p<0.05\right)$. The only Gram-negative bacteria was Pseudomonas spp $(\mathrm{n}=12$; $23.5 \%)$. Amongst the fungal isolates, Fusarium spp was the predominant pathogen detected $\left(n=63 ; 87.5 \% ; \chi^{2}, 142.8\right.$; df, 2 ; $\mathrm{p}<0.0001)$. Other fungi detected were Aspergillus spp and Candida spp. Overall, the commonest cause of microbial keratitis was Fusarium spp ( $\left.\mathrm{n}=63 ; 36.8 \% ; \chi^{2}, 19.20 ; \mathrm{df}, 1 ; \mathrm{p}<0.01\right)$ followed by $S$ aureus $(\mathrm{n}=27 ; 15.8 \%)$ [Figure 3].

Figure 3. Organisms causing keratitis.

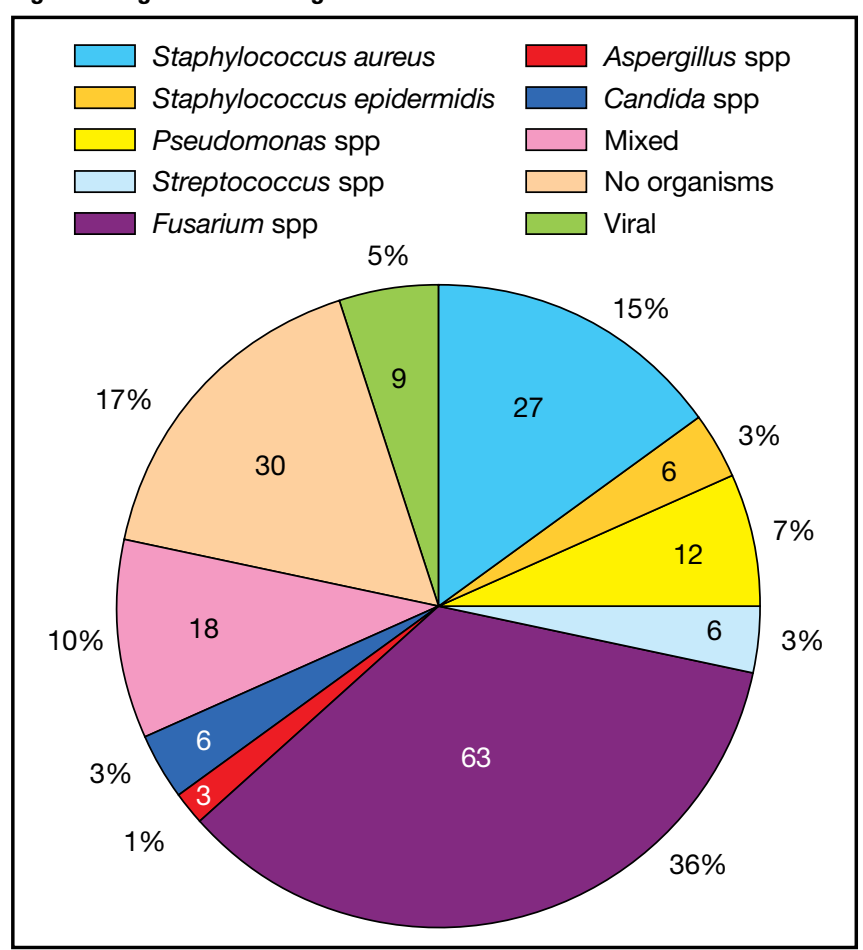


Table 2. Comparison of commonest fungal and bacterial isolates causing microbial keratitis in different regions of India.

\begin{tabular}{|lcccc|}
\hline Region & $\begin{array}{c}\text { Commonest fungal } \\
\text { isolate }\end{array}$ & $\begin{array}{c}\text { Proportion of } \\
\text { fungal cases (\%) }\end{array}$ & $\begin{array}{c}\text { Commonest bacterial } \\
\text { isolate }\end{array}$ & $\begin{array}{c}\text { Proportion of } \\
\text { bacterial cases (\%) }\end{array}$ \\
\hline East India $^{4}$ & Aspergillus $\mathrm{spp}$ & 59.8 & Staphylococcus aureus & 42.6 \\
South India $^{8}$ & Fusarium $\mathrm{spp}$ & 36.6 & Staphylococcus epidermidis & 42.3 \\
South India & Fusarium $\mathrm{spp}$ & 41.9 & Streptococcus pneumoniae & 35.9 \\
North India (present study) & Fusarium $\mathrm{spp}$ & 87.5 & Staphylococcus aureus & 53.0 \\
\hline
\end{tabular}

\section{Discussion}

There are several reports from different countries describing the prevalence of bacterial, fungal, and parasitic pathogens in ulcerated corneas. ${ }^{3-10}$ The epidemiological patterns of microbial keratitis vary between different countries and different geographical areas within a country. Comprehensive local data are therefore important for developing appropriate diagnostic and therapeutic strategies suitable for a particular region.

A significant male preponderance, common occurrence of infectious keratitis in the third to sixth decades of life, and a strong association with previous ocular trauma has been reported. ${ }^{8,12}$ In the present study, most patients $(80 \%)$ were men and $68 \%$ were in the 20-60 years age group. An agricultural occupation and prior trauma with vegetative matter, especially sugarcane leaf, were associated with a significant risk of developing corneal ulceration. These findings are representative of the working habits of the region, where sugarcane farming is carried out on a large scale and a large proportion of the male population in the active years of life is involved in agricultural activities, increasing the likelihood of trauma with vegetative matter.

In this study, the maximum number of corneal ulcers were seen between July and August, when the climate is hot and humid and favours microbial multiplication. A study from southern India has reported similar findings, with the incidence of fungal keratitis being higher between June and September..$^{13}$

The culture positivity rate of $82.5 \%$ in this study was higher than that reported by Basak et $\mathrm{al}^{4}$ of $67.7 \%$ and Gopinathan et $\mathrm{al}^{8}$ of $60.4 \%$. The relatively higher yield in this series may be due to the fact that the specimens obtained from corneal scrapings were not transported to the laboratory, and were directly inoculated into the culture media in the outpatients department. Gram staining and $\mathrm{KOH}$ wet mount smears were able to detect $76.5 \%$ of culturepositive bacterial specimens and $62.5 \%$ of culture-positive fungal specimens. Thus, this method remains useful for the early detection of the causative organism and consequent rapid initiation of treatment, especially in places where facilities for culture are not available.

Fusarium species was the commonest pathogen detected, accounting for $36.8 \%$ of all cases and $87.5 \%$ of the fungal cases. The commonest bacterial pathogen detected was $S$ aureus $(52.9 \%)$ followed by Pseudomonas spp (23.5\%), S epidermidis (11.8\%), and Streptococci spp (11.8\%) of the bacterial cases. A comparison with the results of other studies from southern India ${ }^{8,13}$ and eastern India ${ }^{4}$ (Table 2) shows that the profile of the organisms causing microbial keratitis in the region studied is distinct.

Male sex, agricultural work, and prior injury, especially with sugarcane leaf and other vegetative matter, along with hot and humid weather, are risk factors the development of microbial corneal ulcer in this sample population of the North Indian state of Uttarakhand. Fungal keratitis is more common than bacterial keratitis, with Fusarium spp being the commonest fungus detected. Bacterial keratitis is more commonly caused by Grampositive bacteria, especially $S$ aureus. Knowledge of the local aetiological profile of microbial corneal ulcers will be of value in the management of these patients, especially in remote areas where diagnostic facilities are not easily available and empirical treatment often needs to be started quickly.

\section{References}

1. Whitcher JP, Srinivasan. Corneal ulceration in the developing world - a silent epidemic. Br J Ophthalmol. 1997;81:622-3.

2. Thylefors B, Negrel AD, Pararaja Segaram R, Dadzie KY. Available data on blindness (update 1994). Ophthalmic Epidemiol. 1995;2: 5-39.

3. Williams G, McClellen K, Billson F. Suppurative keratitis in rural Bangladesh: the value of gram stain in planning management. Int Ophthalmol. 1991;15:131-5.

4. Basak SK, Basak S, Mohanta A, Bhowmick A. Epidemiological and microbiological diagnosis of suppurative keratitis in Gangetic West Bengal, eastern India. Indian J Ophthalmol. 2005;53:17-22.

5. Liesegang TJ, Forster RK. Spectrum of microbial keratitis in south Florida. Am J Ophthalmol. 1980;90:38-47.

6. Ormerod LD, Hertzmark E, Gomez DS, et al. Epidemiology of microbial keratitis in southern California. A multivariate analysis. Ophthalmology. 1987;94:1322-33.

7. Srinivasan M, Gonzales CA, George C, et al. Epidemiology and etiological diagnosis of corneal ulceration in Madurai, south India. Br J Ophthalmol. 1997;81:965-71.

8. Gopinathan U, Sharma S, Garg P, Rao GN. Review of epidemiological features, microbiological diagnosis and treatment outcome of microbial keratitis: experience of over a decade. Indian J Ophthalmol. 2009;57:273-9.

9. Dunlop AA, Wright ED, Howlader SA, et al. Suppurative corneal ulceration in Bangladesh. A study of 142 cases examining the microbiological diagnosis, clinical and epidemiological features of bacterial and fungal keratitis. Aust N Z J Ophthalmol. 1994;22: 105-10.

10. Leck AK, Thomas PA, Hagen M, Kaliamurthy J, Acuaku E, John M. Etiology of suppurative corneal ulcer in Ghana and south India, and 
epidemiology of fungal keratitis. $\mathrm{Br} \mathrm{J}$ Ophthalmol. 2002;86:1211-5.

11. Jones DB. Initial therapy of suspected microbial corneal ulcers. II. Specific antibiotic therapy based on corneal smears. Surv Ophthalmol. 1979;24:97, 105-16.

12. Panda A, Satpathy G, Nayak N, Kumar S, Kumar A. Demographic pattern, predisposing factors and management of ulcerative keratitis: evaluation of one thousand unilateral cases at a tertiary care centre. Clin Exper Ophthalmol. 2007;35:44-50.

13. Bharathi MJ, Ramakrishnan R, Meenakshi R, Padmavathy S, Shivakumar C, Srinivasan M. Microbial keratitis in South India: influence of risk factors, climate, and geographical variation. Ophthalmic Epidemiol. 2007;14:61-9. 